\title{
Effects of dietary saturated fatty acids on serum high density lipoprotein, non-high-density lipoprotein and remnant-cholesterol in the Reading Imperial Surrey Saturated fat Cholesterol Intervention (RISSCI-1) study
}

\author{
H. Ayyad ${ }^{1}$, A. Koutsos ${ }^{2}$, R. Antoni ${ }^{1}$, G. Wong ${ }^{2}$, L. Sellem ${ }^{2}$, E. Ozen ${ }^{2}$, K.G. Jackson ${ }^{2}$, \\ M.D. Robertson ${ }^{1}$, B.A. Fielding ${ }^{1}$, J.A. Lovegrove ${ }^{2}$ and B.A. Griffin ${ }^{1}$ \\ ${ }^{1}$ Department of Nutritional Sciences, University of Surrey, Guildford, Surrey, UK and \\ ${ }^{2}$ Hugh Sinclair Unit of Human Nutrition, University of Reading, Whiteknights, Reading, UK
}

Rationale for the dietary guideline to replace saturated (SFA) with unsaturated fatty acids (UFA) to reduce risk of atherosclerotic cardiovascular disease (ASCVD) rests heavily on the lowering of serum low density lipoprotein cholesterol (LDL-C) ${ }^{(1)}$. However, there is evidence to suggest that this dietary exchange may be associated with favourable effects on other cardiometabolic risk factors, including non-high-density lipoprotein-cholesterol (non-HDL-C), a measure of all atherogenic lipoproteins in serum ${ }^{(2)}$. The aim of this study was to determine the effects of dietary SFA on serum HDL-C, non-HDL-C, and remnant-cholesterol in the RISSCI-1 study.

The RISSCI-1 study was a non-randomised, single-blind, dietary intervention study in which 109 healthy men, aged 30 to 65 y, followed two iso-energetic diets; Diet 1 (High SFA 18\% total energy (TE), UFA 15\%TE, total fat 33\% TE), followed immediately by Diet 2 (lower-SFA 10\%TE, high UFA 24\% TE, total fat 34\% TE) for 4 weeks each. Dietary intake was assessed, and fasted blood samples taken for serum lipid analysis, at baseline and after Diets 1 and 2. Serum total and HDL-C were measured by commercially available assays, whereas LDL-C (total C - (HDL-C - TAG/2.2)), non-HDL-C (total C - HDL-C), and remnant-cholesterol (TC - (LDL-C + HDL-C)) were calculated. Statistical comparisons of the difference between baseline and high SFA versus the high SFA and lower SFA diets were made by paired sample t-test.

There were significant differences in the change in serum HDL-C and non-HDL-C between the two dietary interventions (HDL-C mean difference $0.18 \mathrm{mmol} / \mathrm{L}, 95 \%$ CI $0.12,0.24 \mathrm{mmol} / \mathrm{L}, \mathrm{P}<0.001$; non-HDL-C mean difference $0.73 \mathrm{mmol} / \mathrm{L}, 95 \%$ CI $0.57,0.89$, $\mathrm{P}<0.001)$. The mean difference for the change in both HDL-C and non-HDL-C increased during the high SFA diet $(\mathrm{HDL}-\mathrm{C}+4.8 \%$, non-HDL-C $+5.2 \%$ ), and decreased during the lower SFA (high UFA) diet (HDL-C -7.4\%, non-HDL-C $-13.7 \%$ ). There was no significant difference in the change in serum remnant-cholesterol concentrations between diets.

The similar effects of dietary SFA on both serum HDL-C and non-HDL-C in this study, are consistent with a meta-analysis of intervention trials (1), and a previous report of the effects of this dietary exchange on serum LDL-C from the RISSC-1 study ${ }^{(3)}$, given that LDL-C represents the main component of non-HDL-C. Since dietary-induced changes in HDL-C may not be causally related to protection against $\mathrm{ASCVD}^{(4)}$, the effects of SFA on non-HDL-C are of relatively greater clinical importance. Our findings suggest that the favourable difference in non-HDL-C was driven primarily by changes in serum LDL-C in these healthy, normo-lipidaemic men.

\section{Acknowledgements}

Acknowledgement: The RISSCI-1 study was funded by the Biotechnology \& Biological Sciences Research Council (Grant no: BB/ P010245/1).

\section{References}

1. Mensink RP. World Health Organization, Geneva (2016) [Available at: http://apps.who.int/iris/bitstream/handle/10665/246104/97892541565349eng.pdf].

2. Pinart M, Jeran S, Boeing H, et al. (2021) $J$ Nutr (On-line ahead of print).

3. Koutsos A, Antoni R, Ozen E, et al. (2020) Proc Nutr Soc 79, (OCE1) E6.

4. Hewing B, Moore KJ \& Fisher EA (2012) Circ Res 111, 1117-1120. 